\title{
AN IMPROVED PUBLIC KEY CRYPTOSYSTEM BASED ON CHEBYSHEV CHAOTIC MAP OVER FINITE FIELD
}

\section{${ }^{*}$ Yousif S.Najaf ${ }^{1}$}

\author{
Prof. Maher K. Mahmood ${ }^{2}$
}

1) Electrical Engineering Department, University of Al - Mustansiriya, Bagdad, Iraq

2) Electrical Engineering Department, University of Al - Mustansiriya, Bagdad, Iraq

\begin{abstract}
Public key cryptosystem based on a chaotic map over finite field, depends on what is called Chebyshev map, that has a semi-group property and ability to provide a digital signature. The cryptosystem suggested by Ljupco Kocarev has a weakness in its security since it suffers from the state of repetition, resulting into more than one decryption keys (Multiple keys problem).

This paper suggests an improved public key cryptosystem which does not suffer from the repetition, so as to complicate the process of breaking the system since there is only one key that can decrypt the system. This is done by adding a large prime number to the system. Computer simulation results show that the proposed algorithm succeeds to remove the repetition problem completely.
\end{abstract}

Keywords: Public Key, Cryptosystem, Chaotic Map, multi-key problem.

\section{Introduction}

The first ideas of security of communication was presented by (C. E. Shannon) [1, 2]. (W. Diffie and M.E. Hellman) showed for the first time the ability to transfer information in a secret manner without the need to transfer the secret key [3].

Among the most prominent researchers who used chaos in encryption was Ljupco Kocarev [4]. In 2003, Ljupco Kocarev published a paper [5] which suggests the use of chaos in public encryption based on Chebyshev map, in which he used real numbers $[-1,1]$, his idea was then analyzed by Bergamo et al [6]. Then Kocarev repeated the same idea, but using chaotic map over finite field [7]. In 2010 Xiaofeng Liao, Fei Chen, and Kwok- Wo Wong provided an attack on the public key algorithm based on the polynomial Chebyshev over Finite Field $\mathrm{Z}_{\mathrm{N}}$. They showed that the sequences resulting from Chebyshev polynomials on the $\mathrm{Z}_{\mathrm{N}}$ boundary domain have somehow cyclic distribution [8]. In 2013, Jinhui Sun, Geng Zhao and Xufei Li used the multiply $\mathrm{Ki}$ factor method to improve the Kocarev algorithm, where $\mathrm{Ki}$ is secret, and only shared with participants, to overcome the encrypted text attack only as they indicated [9].

Some researchers use some known traditional functions with chaotic systems to avoid the problem by proposing hybrid systems. In 2018 (Nidal Tahat and Emad Abdullah) used the hash functions in addition to Chebyshev map [10].

In this paper, a new way to solve multiple keys problem is suggested by a modification to Kocarev algorithm. 
The paper is arranged as follows: The second section gives basic information about Chebyshev map and properties. The third section explains the Kocarev algorithm to show repetition problem with a numerical example. Section four introduces the proposed algorithm with the conditions of choosing its parameter and an analysis of the performance of it. Section five gives comparisons between the traditional Kocarev algorithm and the proposed algorithm. Section six gives some notes on the proposed method. Finally in section seven, a conclusion is given.

\section{PRELIMINARIE}

In this section, a Chebyshev map and some of its important characteristics are briefly explained.

\subsection{Chebyshev Map}

Chebyshev polynomial map is defined as:

$$
T_{n}(x)=2 x T_{n-1}(x)-T_{n-2}(x)
$$

Where (n) is the order for the polynomial and

(x) is the variable, with initials

$T_{0}(x)=1$ and $T_{1}(x)=x$, some next orders are:

$$
\begin{array}{ll}
\text { for } n=2, & T_{2}(x)=2 x^{2}-1 \\
\text { for } n=3, & T_{3}(x)=4 x^{3}-3 x \quad \text { and so on }
\end{array}
$$

\section{Some Chebyshev polynomial map properties}

1. Semigroup property i.e.

$$
T_{r}\left(T_{s}(x)\right)=T_{r s}(x)
$$

2. Commute under composition i.e.

$$
T_{s}\left(T_{r}(x)\right)=T_{r}\left(T_{s}(x)\right)
$$

for $x \in[-1,1]$ then $\operatorname{Tn}(x) \in[-1,1]$ $\&$ for $x \in Z$ then $\operatorname{Tn}(x) \in Z$.

\subsection{Modular Chebyshev Polynomials Map}

In order to deal with integers, a simple modification of the basic map is made by adding modN to handle a specific field where the definition of the modular map becomes: -

$Y=T_{n}(x) \bmod N$

Where $\mathrm{x}$ and $\mathrm{Y}$ are less than $\mathrm{N}$.

\section{Some Modular Chebyshev Polynomials map properties}

1. Semigroup property i.e.

$T_{s}\left(T_{r}(x) \bmod N\right) \bmod N=T_{r}\left(T_{s}(x) \bmod N\right) \bmod N$

2. Commute under composition i.e.

$$
T_{s}\left(T_{r}(x) \bmod N\right) \bmod N=T_{s r}(x) \bmod N
$$

3. In this case $\mathrm{Y}=1$

a) If order of polynomial is zero.

b) If $(x=1)$ or $(x=N-1 \& n$ is even $)$.

4. May be $Y=0$, that depends on values of $(\mathrm{n}, \mathrm{x}, \mathrm{N})$ used in algorithm.

\section{Traditional algorithm}

In this section, the public key cryptosystem based on Chebyshev map over finite field proposed by Ljupco Kocarev, is explained.

\subsection{Kocarev's algorithm over finite field}

The algorithm has three stages to send a message from Bob to Alice, these are:

i) Keys Generation $\backslash$ to create the keys, Alice will do the following:

1. Choose a large prime number $\mathrm{N}$ and integer number $\mathrm{x}$ less than $\mathrm{N}$. 
2. Compute $A=T_{S}(x) \bmod N$ where $\mathrm{s}$ is a random integer number less than $\mathrm{N}$.

3. The public key for Alice is $(\mathrm{x}, \mathrm{N}, \mathrm{A})$ and the private key is $\mathrm{s}$.

ii) Encryption process $\backslash$ to send the encrypted text, Bob will:

1. Use the public key for Alice (x, N, A)

2. Choose a random integer $r$ less than $\mathrm{N}$.

3. Represent $m \in[0, \mathrm{~N}-1]$.

4. Calculate $B=T_{r}(x) \bmod N$ and

$$
X=m T_{r}(A) \bmod N
$$

5. Send the cipher text $(B, X)$ to Alice. iii) Decryption \ to restore the original message, Alice will:

1.use cipher text $(\mathrm{B}, \mathrm{N})$ to calculate $T_{s}(B) \bmod N$.

2.Finally,compute $m=\left(X / T_{s}(B) \bmod N\right) \bmod N$

\subsection{Analysis of Kocarev's algorithm and multi- key problem}

The cryptosystem based on Chebyshev map is analyzed by the analysis of the map itself. In order to analyze the map, a table is generated for the results produced from the Chebyshev map. Since the value of $x$ is fixed in the algorithm we will compare the results produced from the same value of $\mathrm{x}$ together i.e. we will compare the results in each row in the table. Table (1) shows the $T_{n}(x) \bmod N$ values for $\mathrm{N}=19$.

Table 1. Chebyshev Map $T_{n}(x) \bmod N$ with $\mathrm{N}=19$

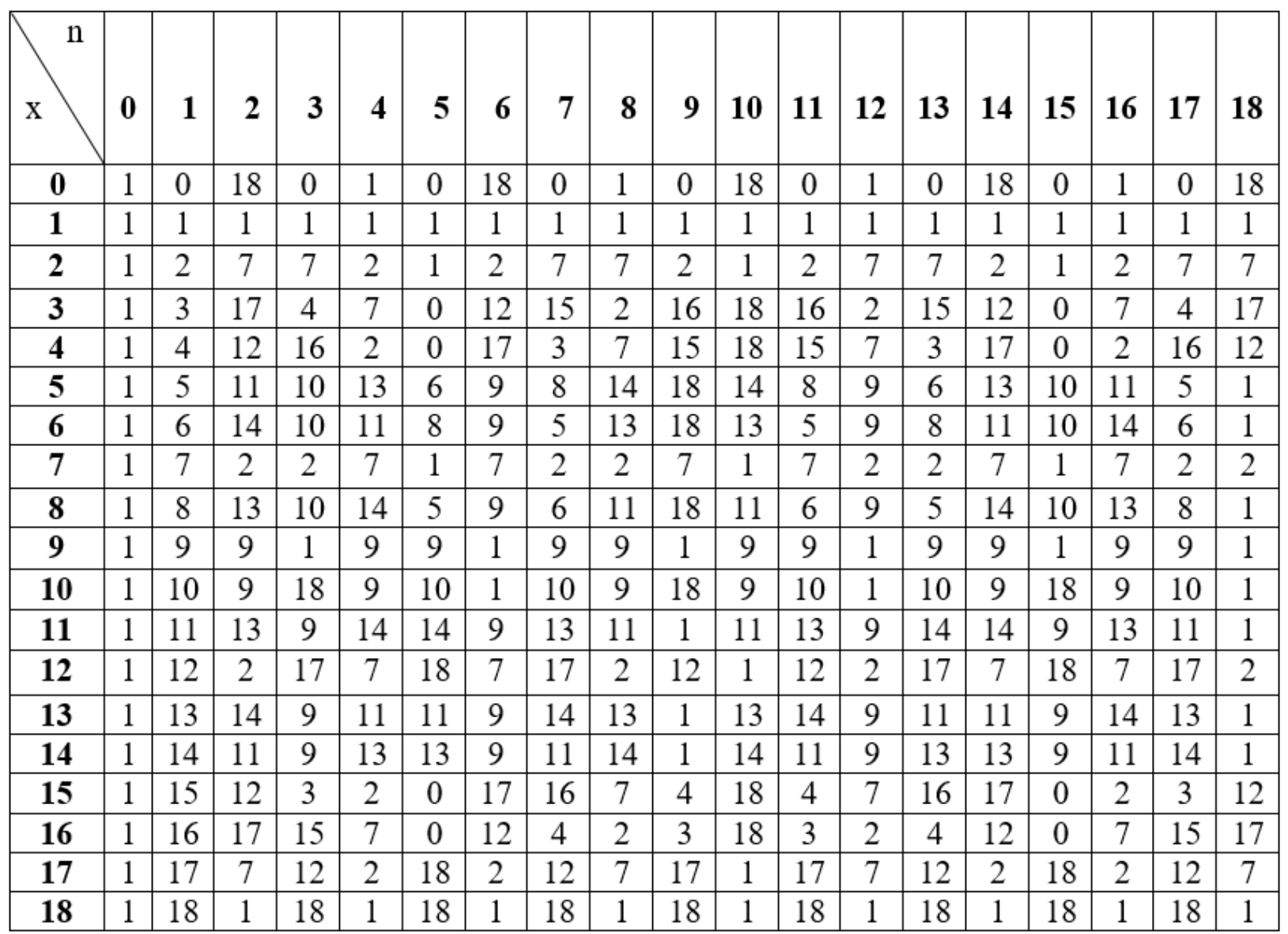


For $(x=6)$ for example then we will see that for $n=(3,15)$ the result is 10 and for $n=(8,10)$ the result is 13 and so on other repetitions are shown in figure (1).

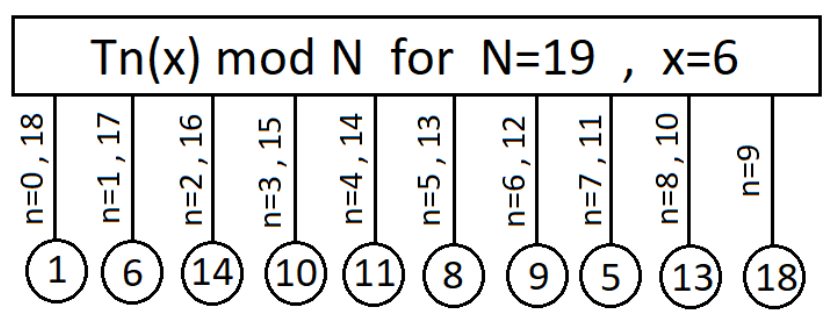

Figure 1. Chebyshev Map $T_{n}(x) \bmod N$ repetitions with $\mathrm{N}=19 \& \mathrm{x}=6$

Similarly, for $\mathrm{x}=11$ then we will see that for $\mathrm{n}=$ $(1,8,10,17)$ the result is $(11)$ and for $n=(3,6$, $12,15)$ the result is (9) and so on as shown in figure (2).

\section{$\operatorname{Tn}(\mathrm{x}) \bmod \mathrm{N}$ for $\mathrm{N}=19, \mathrm{x}=11$

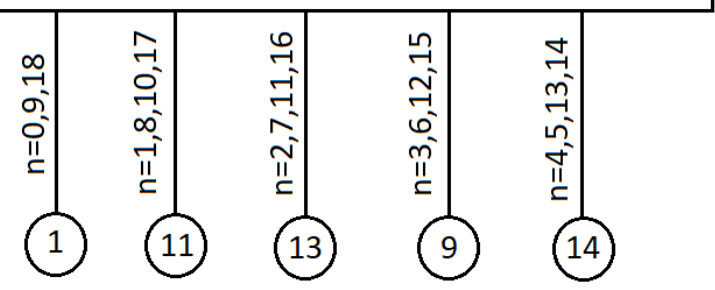

Figure 2. Chebyshev Map $T_{n}(x) \bmod N$ repetition with $\mathrm{N}=19 \& \mathrm{x}=11$

The number of $\mathrm{n}$ that produces the same result from the map is related with the choice of $x$. For example, if $x=6$, then two values of $n$ produce the same result; while for $x=11$ four values of $n$ produce the same results. Therefore, the choice of $x=11$ is worse than the choice of $x=6$.

The important thing is that the repetition problem exists for every values of $(x)$ from $x=0$ to $\mathrm{x}=18$ and not only for $\mathrm{N}=19$ but for any other values for $(\mathrm{N})$, This repetition will produce multiple keys for the same value of $\mathrm{x}$.

\subsection{Encryption/ Decryption example for Kocarev's algorithm}

If Bob sends $m=10$ message to Alice, then the three stages of the algorithm are;

Generating keys $\backslash$ Suppose Alice chooses $\mathrm{N}=$ $19, \mathrm{~s}=8, \mathrm{x}=11$, and then she calculated $A=T_{s}(x) \bmod N=T_{8}(11) \bmod 19=11, \quad$ the public key for Alice is $(11,19,11)$ and the private key is (8).

Encryption process $\backslash$ to send the encrypted text, Bob will use the public key for Alice $(11,19,11)$ then he Chooses $\mathrm{r}=16$, to calculate $B=$ $T_{r}(x) \bmod N=T_{16}(11) \bmod 19=13$ and $X=$ $m \times T_{r}(A) \bmod N=10 \times T_{16}(11) \bmod 19=$ $10 \times 13=130$, finally Bob sends the cipher text to Alice $(B, X)=(13,130)$.

Decryption $\backslash$ message recovery process; Alice uses cipher text $(13,130)$ to calculate $T_{s}(B) \bmod N=T_{8}(13) \bmod 19=13 \quad$, then computes $\quad m=\left(X / T_{s}(B) \bmod N\right) \bmod N=$ $(130 / 13) \bmod 19=10=$ original message $\mathrm{m}$.

Because of the problem of multiple keys, the third party can attack the system easily. Where the value of ( $\mathrm{s}$ ) can be any of the following values $(1,8,10,17)$ and the value of $(r)$ can be any of the following values $(2,7,11,16)$ as shown in figure (2).

\section{The proposed improved public key cryptosystem based on Chebyshev map over finite field}

\subsection{The proposed improved algorithm over finite field}

In the improved algorithm, a large prime number $(\mathrm{P}>\mathrm{N})$ is generated; where $\mathrm{N}$ is not necessarily prime number and where $(\mathrm{s}, \mathrm{r}, \mathrm{m}) \in[0, \mathrm{~N}-1]$. The algorithm has also three stages, these are: 
i) Keys Generation \to create the keys, Alice will do the following:

1.Choose a large integer number $\mathrm{N}$ and an appropriate integer number $\mathrm{x}<\mathrm{N}$.

2. Choose a large prime number $\mathrm{P}>\mathrm{N}$.

3. Compute $A=T_{S}(x) \bmod P$, where s is a random integer number less than $\mathrm{N}$.

4. The public key for Alice is $(x, N, P, A)$ and the private key is $\mathrm{s}$.

ii) Encryption process $\backslash$ to send the encrypted text, Bob will:

1. use the public key for Alice $(x, N, P, A)$.

2. Choose a random integer $\mathrm{r}$ less than $\mathrm{N}$.

3. Represent $m \in[0, \mathrm{~N}-1]$.

4.Calculate $B=T_{r}(x) \bmod P$ and

$X=m \times T_{r}(A) \bmod P$.

5. Send the cipher text to Alice $(B, X)$.

iii) Decryption $\backslash$ to restore the original message, Alice will:

1. use cipher text $(B, X)$ to calculate $T_{S}(B) \bmod P$.

2.Finally compute $m=\left(X / T_{s}(B) \bmod P\right) \bmod P$

\subsection{How to choose an appropriate value for $(P)$}

It is important to choose an appropriate value for (P) to solve the problem of multiple keys. To achieve that, $(\mathrm{P})$ is chosen as a large prime number and the following conditions are satisfied:

1-The obtained outputs are not similar to each other for a specific value of $(x)$ for all possible values of $(\mathrm{n})$ since the value of $(\mathrm{x})$ is fixed in the algorithm, hence $x \in[2, \mathrm{~N}-1]$.
2- The obtained outputs are not equal to (1) such that the cipher text is not equal to plain text. This will exclude $n=0 \& x=1$.

3- The obtained outputs are not equal to (P-1) because the value of $\left(T_{n}(P-1) \bmod P=1\right)$ for even value of (n) and hence the cipher text equals to plain text $\left(X=m \times T_{r}(A) \bmod P=\right.$ $m)$.

4- The obtained outputs are not equal to (0) because the value of $\left(T_{n}(0) \bmod P\right)$ equals to one of three output $(0,1, \mathrm{P}-1)$ for any value of (n) and hence if the output is (0) the plain text cannot be restored.

The first condition makes the range of possible value of $x \in[2, \mathrm{~N}-1]$ and the second condition makes the range of possible value of $n \in[1, \mathrm{~N}-1]$.

As a conclusion from above, the value of ( $\mathrm{x}$ ) must produce $(\mathrm{N}-1)$ different outputs and does not include any output equal to $(0,1$, and $\mathrm{P}-1)$ from the map $\left(T_{n}(x) \bmod P\right)$ for a certain value of $(\mathrm{P})$ and for all orders $n=[1, N-1]$.

For any value of $(\mathrm{P})$ there are certain values of (x) that can be used to satisfy the conditions. The number of $(\mathrm{x})$ values that satisfies the conditions is shown to be related with the ratio $(\mathrm{P} / \mathrm{N})$. Where increasing the ratio of $(\mathrm{P} / \mathrm{N})$ leading to increase the number of $(\mathrm{x})$ that can meet the required conditions. More values of possible (x) may satisfy the conditions when the ratio $(\mathrm{P} / \mathrm{N})$ is large enough.

\subsection{Analysis of the performance of the proposed improved algorithm}

To analyze the map, the results produced from the modified Chebyshev map is generated in a table since the value of ( $\mathrm{x}$ ) is fixed in algorithm. Table (2) shows the results for $\mathrm{N}=19, \mathrm{P}=41$. 
Table 2. Modified Chebyshev Map $T_{n}(x) \bmod N$ with $\mathrm{N}=19, \mathrm{P}=41$

\begin{tabular}{|c|c|c|c|c|c|c|c|c|c|c|c|c|c|c|c|c|c|c|c|}
\hline $\mathbf{n}$ & & & & & & & & & & & & & & & & & & & \\
& $\mathbf{0}$ & $\mathbf{l}$ & $\mathbf{2}$ & $\mathbf{3}$ & $\mathbf{4}$ & $\mathbf{5}$ & $\mathbf{6}$ & $\mathbf{7}$ & $\mathbf{8}$ & $\mathbf{9}$ & $\mathbf{1 0}$ & $\mathbf{1 1}$ & $\mathbf{1 2}$ & $\mathbf{1 3}$ & $\mathbf{1 4}$ & $\mathbf{1 5}$ & $\mathbf{1 6}$ & $\mathbf{1 7}$ & $\mathbf{1 8}$ \\
\hline $\mathbf{0}$ & 1 & 0 & 40 & 0 & 1 & 0 & 40 & 0 & 1 & 0 & 40 & 0 & 1 & 0 & 40 & 0 & 1 & 0 & 40 \\
\hline $\mathbf{1}$ & 1 & 1 & 1 & 1 & 1 & 1 & 1 & 1 & 1 & 1 & 1 & 1 & 1 & 1 & 1 & 1 & 1 & 1 & 1 \\
\hline $\mathbf{2}$ & 1 & 2 & 7 & 26 & 15 & 34 & 39 & 40 & 39 & 34 & 15 & 26 & 7 & 2 & 1 & 2 & 7 & 26 & 15 \\
\hline $\mathbf{3}$ & 1 & 3 & 17 & 17 & 3 & 1 & 3 & 17 & 17 & 3 & 1 & 3 & 17 & 17 & 3 & 1 & 3 & 17 & 17 \\
\hline $\mathbf{4}$ & 1 & 4 & 31 & 39 & 35 & 36 & 7 & 20 & 30 & 15 & 8 & 8 & 15 & 30 & 20 & 7 & 36 & 35 & 39 \\
\hline $\mathbf{5}$ & 1 & 5 & 8 & 34 & 4 & 6 & 15 & 21 & 31 & 2 & 30 & 11 & 39 & 10 & 20 & 26 & 35 & 37 & 7 \\
\hline $\mathbf{6}$ & 1 & 6 & 30 & 26 & 36 & 37 & 39 & 21 & 8 & 34 & 31 & 10 & 7 & 33 & 20 & 2 & 4 & 5 & 15 \\
\hline $\mathbf{7}$ & 1 & 7 & 15 & 39 & 39 & 15 & 7 & 1 & 7 & 15 & 39 & 39 & 15 & 7 & 1 & 7 & 15 & 39 & 39 \\
\hline $\mathbf{8}$ & 1 & 8 & 4 & 15 & 31 & 30 & 39 & 20 & 35 & 7 & 36 & 36 & 7 & 35 & 20 & 39 & 30 & 31 & 15 \\
\hline $\mathbf{9}$ & 1 & 9 & 38 & 19 & 17 & 0 & 24 & 22 & 3 & 32 & 40 & 32 & 3 & 22 & 24 & 0 & 17 & 19 & 38 \\
\hline $\mathbf{1 0}$ & 1 & 10 & 35 & 34 & 30 & 33 & 15 & 21 & 36 & 2 & 4 & 37 & 39 & 5 & 20 & 26 & 8 & 11 & 7 \\
\hline $\mathbf{1 1}$ & 1 & 11 & 36 & 2 & 8 & 10 & 7 & 21 & 4 & 26 & 35 & 6 & 15 & 37 & 20 & 34 & 31 & 33 & 39 \\
\hline $\mathbf{1 2}$ & 1 & 12 & 0 & 29 & 40 & 29 & 0 & 12 & 1 & 12 & 0 & 29 & 40 & 29 & 0 & 12 & 1 & 12 & 0 \\
\hline $\mathbf{1 3}$ & 1 & 13 & 9 & 16 & 38 & 29 & 19 & 14 & 17 & 18 & 0 & 23 & 24 & 27 & 22 & 12 & 3 & 25 & 32 \\
\hline $\mathbf{1 4}$ & 1 & 14 & 22 & 28 & 24 & 29 & 9 & 18 & 3 & 25 & 0 & 16 & 38 & 23 & 32 & 12 & 17 & 13 & 19 \\
\hline $\mathbf{1 5}$ & 1 & 15 & 39 & 7 & 7 & 39 & 15 & 1 & 15 & 39 & 7 & 7 & 39 & 15 & 1 & 15 & 39 & 7 & 7 \\
\hline $\mathbf{1 6}$ & 1 & 16 & 19 & 18 & 24 & 12 & 32 & 28 & 3 & 27 & 0 & 14 & 38 & 13 & 9 & 29 & 17 & 23 & 22 \\
\hline $\mathbf{1 7}$ & 1 & 17 & 3 & 3 & 17 & 1 & 17 & 3 & 3 & 17 & 1 & 17 & 3 & 3 & 17 & 1 & 17 & 3 & 3 \\
\hline $\mathbf{1 8}$ & 1 & 18 & 32 & 27 & 38 & 29 & 22 & 25 & 17 & 13 & 0 & 28 & 24 & 16 & 19 & 12 & 3 & 14 & 9 \\
\hline
\end{tabular}

When we notice the outputs from the map for ( $\mathrm{x}$ $=6$ ) in table (2), we get (18) different outputs from the modified Chebyshev map corresponding to $\mathrm{n}=1 \rightarrow 18$ as shown in figure (3).

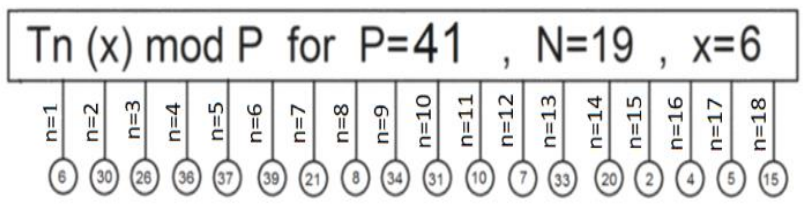

Figure 3. Modified Chebyshev Map $T_{n}(x) \bmod P$ with $\mathrm{P}=41, \mathrm{~N}=19, \mathrm{x}=6$

The same thing will be observed for values of $\mathrm{x}=\{5,10,11\}$, the map produces (18) different outputs. Figure (4) shows the outputs of modified Chebyshev map when $\mathrm{x}=11$.

\section{Tn $(\mathrm{x}) \bmod \mathrm{P}$ for $\mathrm{P}=41, \mathrm{~N}=19, \mathrm{x}=11$ \\ त)

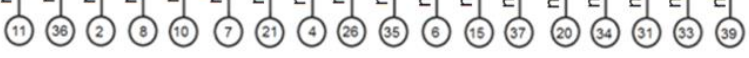

Figure 4. Modified Chebyshev Map $T_{n}(x) \bmod P$ with $\mathrm{P}=41, \mathrm{~N}=19, \mathrm{x}=11$

But, if we notice the outputs from modified Chebyshev map for $\mathrm{x}=9$ we get only (10) different outputs i.e. repetition state still exists and the output includes $(0,40)$ as shown in Figure (5).

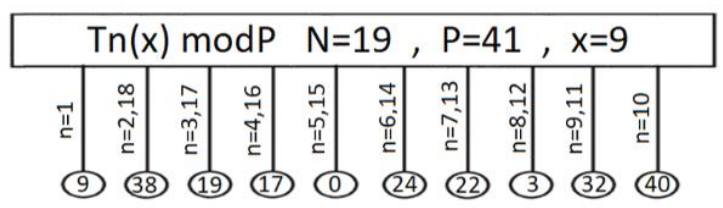

Figure 5. Modified Chebyshev Map $T_{n}(x) \bmod P$ with $\mathrm{P}=41, \mathrm{~N}=19, \mathrm{x}=9$

The same thing will be observed for values of $\mathrm{x}=\{0,1,2,3,4,7,8,12,13,14,15,16,17,18\}$ the problem of repetition still exists.

Thus $\left(T_{n}(x) \bmod P\right)$ can overcome the problem of repetition (multiple keys) for $\mathrm{x}=\{5,6,10,11\}$, but it fails for $\mathrm{x}=\{0,1,2,3,4,7,8,9,12,13,14$, $15,16,17,18\}$.

As shown above, choosing the value of $x$ is very important and in order to increase the number of (x) values satisfying the conditions, we increase the ratio $(\mathrm{P} / \mathrm{N})$ say $(\mathrm{P} / \mathrm{N}>20)$. For example, if we choosing $\mathrm{N}=19, \mathrm{P}=383$ and computing $\left(T_{n}(x) \bmod P\right)$, table (3) is produced. 
Table 3. Modified Chebyshev Map $T_{n}(x) \bmod N$ with $\mathrm{N}=19, \mathrm{P}=383$

\begin{tabular}{|c|l|l|l|l|l|l|l|l|l|l|l|l|l|l|l|l|l|l|l|}
\hline $\mathbf{n}$ & & & & & & & & & & & & & & & & & & & \\
& $\mathbf{0}$ & $\mathbf{l}$ & $\mathbf{2}$ & $\mathbf{3}$ & $\mathbf{4}$ & $\mathbf{5}$ & $\mathbf{6}$ & $\mathbf{7}$ & $\mathbf{8}$ & $\mathbf{9}$ & $\mathbf{1 0}$ & $\mathbf{1 1}$ & $\mathbf{1 2}$ & $\mathbf{1 3}$ & $\mathbf{1 4}$ & $\mathbf{1 5}$ & $\mathbf{1 6}$ & $\mathbf{1 7}$ & $\mathbf{1 8}$ \\
\hline $\mathbf{0}$ & 1 & 0 & 382 & 0 & 1 & 0 & 382 & 0 & 1 & 0 & 382 & 0 & 1 & 0 & 382 & 0 & 1 & 0 & 382 \\
\hline $\mathbf{1}$ & 1 & 1 & 1 & 1 & 1 & 1 & 1 & 1 & 1 & 1 & 1 & 1 & 1 & 1 & 1 & 1 & 1 & 1 & 1 \\
\hline $\mathbf{2}$ & 1 & 2 & 7 & 26 & 97 & 362 & 202 & 63 & 50 & 137 & 115 & 323 & 28 & 172 & 277 & 170 & 20 & 293 & 3 \\
\hline $\mathbf{3}$ & 1 & 3 & 17 & 99 & 194 & 299 & 68 & 109 & 203 & 343 & 323 & 63 & 55 & 267 & 15 & 206 & 72 & 226 & 135 \\
\hline $\mathbf{4}$ & 1 & 4 & 31 & 244 & 6 & 187 & 341 & 243 & 71 & 325 & 231 & 374 & 80 & 266 & 133 & 32 & 123 & 186 & 216 \\
\hline $\mathbf{5}$ & 1 & 5 & 49 & 102 & 205 & 33 & 125 & 68 & 172 & 120 & 262 & 202 & 226 & 143 & 55 & 24 & 185 & 294 & 74 \\
\hline $\mathbf{6}$ & 1 & 6 & 71 & 80 & 123 & 247 & 160 & 141 & 0 & 242 & 223 & 136 & 260 & 303 & 312 & 377 & 382 & 377 & 312 \\
\hline $\mathbf{7}$ & 1 & 7 & 97 & 202 & 50 & 115 & 28 & 277 & 20 & 3 & 22 & 305 & 35 & 185 & 257 & 349 & 33 & 113 & 17 \\
\hline $\mathbf{8}$ & 1 & 8 & 127 & 109 & 85 & 102 & 15 & 138 & 278 & 97 & 125 & 371 & 66 & 302 & 170 & 120 & 218 & 304 & 50 \\
\hline $\mathbf{9}$ & 1 & 9 & 161 & 208 & 136 & 325 & 352 & 266 & 223 & 301 & 216 & 140 & 6 & 351 & 184 & 280 & 260 & 187 & 42 \\
\hline $\mathbf{1 0}$ & 1 & 10 & 199 & 140 & 303 & 175 & 133 & 187 & 160 & 332 & 352 & 197 & 141 & 325 & 231 & 82 & 260 & 139 & 222 \\
\hline $\mathbf{1 1}$ & 1 & 11 & 241 & 312 & 112 & 237 & 123 & 171 & 192 & 223 & 118 & 75 & 0 & 308 & 265 & 160 & 191 & 212 & 260 \\
\hline $\mathbf{1 2}$ & 1 & 12 & 287 & 365 & 47 & 380 & 264 & 211 & 204 & 89 & 17 & 319 & 362 & 326 & 185 & 284 & 120 & 298 & 138 \\
\hline $\mathbf{1 3}$ & 1 & 13 & 337 & 323 & 18 & 145 & 305 & 125 & 264 & 228 & 302 & 347 & 294 & 20 & 226 & 111 & 362 & 109 & 174 \\
\hline $\mathbf{1 4}$ & 1 & 14 & 8 & 210 & 127 & 282 & 109 & 89 & 85 & 376 & 102 & 182 & 15 & 238 & 138 & 179 & 278 & 328 & 97 \\
\hline $\mathbf{1 5}$ & 1 & 15 & 66 & 50 & 285 & 74 & 20 & 143 & 57 & 35 & 227 & 264 & 33 & 343 & 299 & 201 & 369 & 145 & 151 \\
\hline $\mathbf{1 6}$ & 1 & 16 & 128 & 250 & 212 & 23 & 141 & 276 & 265 & 161 & 291 & 342 & 312 & 67 & 300 & 341 & 271 & 288 & 136 \\
\hline $\mathbf{1 7}$ & 1 & 17 & 194 & 68 & 203 & 323 & 55 & 15 & 72 & 135 & 305 & 277 & 304 & 101 & 66 & 228 & 26 & 273 & 64 \\
\hline $\mathbf{1 8}$ & 1 & 18 & 264 & 294 & 362 & 99 & 138 & 273 & 115 & 37 & 68 & 113 & 170 & 262 & 70 & 343 & 22 & 66 & 56 \\
\hline
\end{tabular}

In table (3), all $x$ values are usable because they meet the required conditions except $x=6$ and $\mathrm{x}=11$ in addition to zero and one that does not satisfy the conditions. For $x=6$ the map produces (16) different outputs and for $x=11$ the map produces (18) different output but one of these output is zero. The outputs for $\mathrm{x}=6$ and $\mathrm{x}=11$ are shown in figure (6).

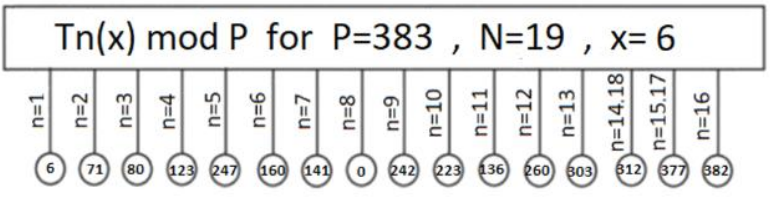

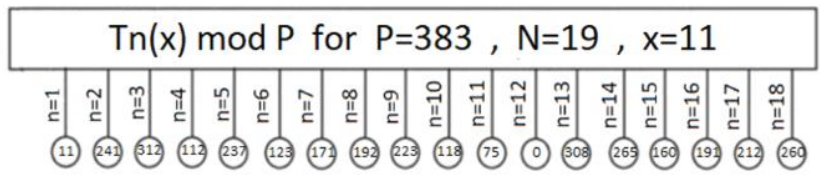

Figure 6. Modified Chebyshev Map $T_{n}(x) \bmod P$ with $\mathrm{P}=383, \mathrm{~N}=19, \mathrm{x}=6,11$

Consequently, the improved algorithm can overcome the problem of multiple keys, whether by using the first or second ratio, but the difference between them is the number of $\mathrm{x}$ values satisfying the conditions, where in the $((\mathrm{P} / \mathrm{N})>20)$ ratio (15) values of $(\mathrm{x})$ satisfy the conditions but, for $((\mathrm{P} / \mathrm{N})>2)$ only $(4)$ values of (x) satisfy the conditions.

To clarify the previous relationships between the number of $(\mathrm{x})$ values satisfying the conditions and the ratio $(\mathrm{P} / \mathrm{N})$ let us assume that:

- (M) represents the number of $(\mathrm{x})$ values that meet the required conditions.

- (Z) represents the number of all possible values of $(\mathrm{x})$ which is equal to $(\mathrm{N}-2)$.

- (Q) Represents the ratio of the number of $\mathrm{x}$ values that fulfill the conditions to the number of all possible $x$ value, i.e. $\mathrm{Q}=(\mathrm{M} / \mathrm{Z}) 100 \%$.

The values of $(\mathrm{Z}, \mathrm{P}, \mathrm{M}, \mathrm{Q})$ are calculated in the table (4) for the ratio $(\mathrm{P} / \mathrm{N})$ greater than $(2,20$, 200 ) and for the range of values of $\mathrm{N}=[10,40]$ to show the effect of the ratio $(\mathrm{P} / \mathrm{N})$ on the number of $(\mathrm{x})$ values that meet the condition $(\mathrm{M})$. 
Table 4. The values of $(\mathrm{Z}, \mathrm{P}, \mathrm{M}, \mathrm{Q})$ with $\mathrm{N}=[10,40]$

\begin{tabular}{|c|c|c|c|c|c|c|c|c|c|c|}
\hline $\mathrm{N}$ & $\mathrm{Z}$ & $\begin{array}{c}\mathrm{P} \\
[\mathrm{P} N)>2]\end{array}$ & $\begin{array}{c}\mathrm{M} \\
{[(\mathrm{P} N)>2]}\end{array}$ & $\begin{array}{c}\mathrm{Q} \% \\
[\mathrm{P} / \mathrm{N})>2]\end{array}$ & $\begin{array}{c}\mathrm{P} \\
{[(\mathrm{P} N)>20]}\end{array}$ & $\begin{array}{c}\mathrm{M} \\
[\mathrm{P} / \mathrm{N})>20]\end{array}$ & $\begin{array}{c}\mathrm{Q} \% \\
[\mathrm{P} N)>20]\end{array}$ & $\begin{array}{c}\mathrm{P} \\
{[(\mathrm{P} N)>200]}\end{array}$ & $\begin{array}{c}\mathrm{M} \\
[\mathrm{P} / \mathrm{P})>200]\end{array}$ & $\left.\begin{array}{c}\mathrm{Q} \% \\
[\mathrm{P} / \mathrm{N}) 200]\end{array}\right]$ \\
\hline 10 & 8 & 23 & 1 & 12.5 & 211 & 7 & 87.5 & 2003 & 8 & 100 \\
\hline 11 & 9 & 23 & 1 & 11.11 & 223 & 8 & 88.88 & 2203 & 9 & 100 \\
\hline 12 & 10 & 29 & 1 & 10 & 241 & 6 & 60 & 2411 & 10 & 100 \\
\hline 13 & 11 & 29 & 1 & 9.09 & 263 & 11 & 100 & 2609 & 11 & 100 \\
\hline 14 & 12 & 29 & 1 & 8.33 & 281 & 11 & 91.66 & 2801 & 12 & 100 \\
\hline 15 & 13 & 31 & 2 & 15.38 & 307 & 10 & 76.92 & 3001 & 13 & 100 \\
\hline 16 & 14 & 37 & 4 & 28.57 & 331 & 14 & 100 & 3203 & 14 & 100 \\
\hline 17 & 15 & 37 & 4 & 26.66 & 347 & 14 & 93.33 & 3407 & 15 & 100 \\
\hline 18 & 16 & 37 & 4 & 25 & 367 & 15 & 93.75 & 3607 & 16 & 100 \\
\hline 19 & 17 & 41 & 4 & 23.52 & 383 & 15 & 88.23 & 3803 & 17 & 100 \\
\hline 20 & 18 & 41 & 4 & 22.22 & 401 & 17 & 94.44 & 4001 & 18 & 100 \\
\hline 21 & 19 & 43 & 4 & 21.05 & 421 & 17 & 89.47 & 4201 & 19 & 100 \\
\hline 22 & 20 & 47 & 3 & 15 & 443 & 17 & 85 & 4409 & 20 & 100 \\
\hline 23 & 21 & 47 & 3 & 14.28 & 461 & 18 & 85.71 & 4603 & 21 & 100 \\
\hline 24 & 22 & 53 & 4 & 18.18 & 487 & 21 & 95.45 & 4801 & 21 & 95.45 \\
\hline 25 & 23 & 53 & 5 & 21.79 & 503 & 20 & 86.95 & 5003 & 22 & 95.65 \\
\hline 26 & 24 & 53 & 5 & 20.83 & 521 & 19 & 79.16 & 5209 & 24 & 100 \\
\hline 27 & 25 & 59 & 9 & 36 & 541 & 24 & 96 & 5407 & 24 & 96 \\
\hline 28 & 26 & 59 & 10 & 38.46 & 563 & 26 & 100 & 5623 & 26 & 100 \\
\hline 29 & 27 & 59 & 11 & 40.74 & 587 & 23 & 85.18 & 5801 & 27 & 100 \\
\hline 30 & 28 & 61 & 7 & 25 & 601 & 21 & 75 & 6007 & 28 & 100 \\
\hline 31 & 29 & 67 & 6 & 20.68 & 631 & 28 & 96.55 & 6203 & 29 & 100 \\
\hline 32 & 30 & 67 & 6 & 20 & 641 & 25 & 83.33 & 6421 & 29 & 96.66 \\
\hline 33 & 31 & 67 & 6 & 19.35 & 661 & 27 & 87.09 & 6607 & 30 & 96.77 \\
\hline 34 & 32 & 71 & 4 & 12.5 & 683 & 28 & 87.5 & 6803 & 31 & 96.87 \\
\hline 35 & 33 & 71 & 5 & 15.15 & 701 & 29 & 87.87 & 7001 & 33 & 100 \\
\hline 36 & 34 & 73 & 9 & 26.47 & 727 & 32 & 94.11 & 7207 & 34 & 100 \\
\hline 37 & 35 & 79 & 5 & 14.28 & 743 & 33 & 94.28 & 7411 & 34 & 97.14 \\
\hline 38 & 36 & 79 & 5 & 13.88 & 761 & 33 & 91.66 & 7603 & 36 & 100 \\
\hline 39 & 37 & 79 & 6 & 16.21 & 787 & 37 & 100 & 7817 & 37 & 100 \\
\hline 40 & 38 & 83 & 14 & 36.84 & 809 & 35 & 92.1 & 8009 & 38 & 100 \\
\hline & Average ratio & & $20.61 \%$ & & & $89.26 \%$ & & & $99.17 \%$ \\
\hline
\end{tabular}

From table (4) we find that increasing the ratio $(\mathrm{P} / \mathrm{N})$ leads to increase the value of $(\mathrm{M})$ and hence the value of $(\mathrm{Q})$ will increase.

The Q results of table (4) are plotted in figure 7.

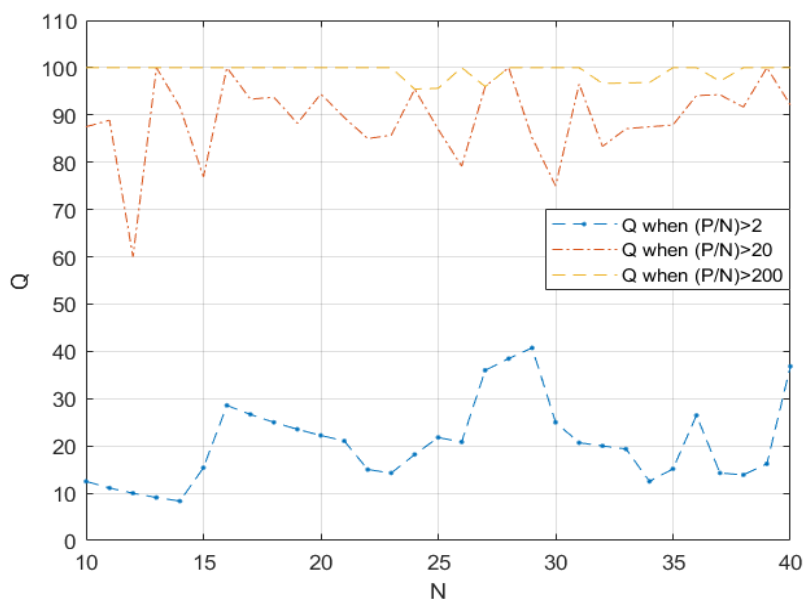

Figure 7. The relationship between $(\mathrm{Q}, \mathrm{N})$

\subsection{Example of the proposed algorithm}

Suppose Bob wants to send $\mathrm{m}=10$ message to Alice, where the three stages of the algorithm are:
Generating keys $\backslash$ Suppose Alice chooses $\mathrm{N}=19$, $\mathrm{P}=41, \mathrm{~s}=8, \mathrm{x}=11$, and then calculated $A=T_{s}(x) \bmod P=T_{8}(11) \bmod 41=4$, the public key for Alice is $(11,19,41,4)$ and private key is (8).

Encryption process $\backslash$ to send the encrypted text, Bob will use the public key for Alice is $(11,19$, $41,4)$ then Choose $r=16, m=10$ and Calculate $B=T_{r}(x) \bmod P=T_{16}(11) \bmod 41=31$ and $X=m \times T_{r}(A) \bmod P=10 \times T_{16}(4) \bmod 41$ $=10 \times 36=360$, finally Bob sends the cipher text to Alice $(31,360)$.

Decryption \to restore the original message. Alice will use cipher text $(31,360)$ to calculate $T_{S}(\boldsymbol{B}) \bmod P=T_{8}(31) \bmod 41=36, \quad$ then computes $m=\left(X / T_{S}(B) \bmod P\right) \bmod P=(360 /$ $36) \bmod 41=10$ equal to original message. There is only one value for $(s, r)$ in order to decrypt the system the third party must know. This makes the attack more difficult. 


\section{Comparison between the traditional algorithm and the proposed algorithm}

In this section, the traditional Kocarev algorithm is compared with the proposed algorithm, where we will use three ratios $(\mathrm{P} / \mathrm{N})$ with the proposed algorithm $((\mathrm{P} / \mathrm{N})>2,(\mathrm{P} / \mathrm{N})>20,(\mathrm{P} / \mathrm{N})>200)$. The comparison is made based on the ratio of the number of $\mathrm{x}$ values that fulfill the conditions to the number of all possible $\mathrm{x}$ values that can be used, hence:

-Take the values of $\mathrm{N}$ as those prime number in the range $5 \rightarrow 97$ since this condition is required in the traditional algorithm. Table (5) shows a comparison between the proposed \& traditional algorithms in term of (Z, P, M, Q).
When observing the above table, the value of $(\mathrm{M})$ of the traditional algorithm equal to zero for all values of $(\mathrm{N})$. This indicates that the traditional Kocarev algorithm suffers from the problem of multiple keys of all values of $(\mathrm{N})$.

However, the values of $(\mathrm{Q})$ of the proposed algorithm indicated that the proposed method solves the problem of multiple keys. In the proposed algorithm, the ratio $(\mathrm{P} / \mathrm{N})>2)$ succeeds to solve the problem of multiple keys for $23.51 \%$ of the values of $(\mathrm{x})$. The ratio $(\mathrm{P} / \mathrm{N})>20)$ succeeds to solve the problem for $91.91 \%$ of the values of $(\mathrm{x})$. The ratio $(\mathrm{P} / \mathrm{N})>200)$ succeeds to solve the problem for $99.23 \%$ of the values of (x). The $\mathrm{Q}$ values are plotted against $\mathrm{N}$ as shown in Figure (8).

Table 5. Comparison between the proposed \& traditional algorithms

\begin{tabular}{|c|c|c|c|c|c|c|c|c|c|c|c|c|}
\hline $\mathrm{N}$ & $z$ & $\begin{array}{c}\mathrm{M} \\
\text { for } \\
\text { traditional } \\
\text { algorithm }\end{array}$ & $\begin{array}{c}\text { Q } \\
\text { for } \\
\text { traditional } \\
\text { algorithm }\end{array}$ & $\begin{array}{c}\text { p } \\
\text { where } \\
(\mathrm{P} N)>2)\end{array}$ & $\begin{array}{l}\mathrm{M} \\
\text { For } \\
\text { proposed } \\
\text { algorithm } \\
\text { Where } \\
(\mathrm{P} / \mathrm{N})>2 \text { ) }\end{array}$ & \begin{tabular}{c|}
$\mathrm{Q}$ \\
For \\
proposed \\
algorithm \\
Where \\
$(\mathrm{P} / \mathrm{N})>2)$
\end{tabular} & $\begin{array}{c}\text { P } \\
\text { where } \\
(\mathrm{P} / \mathrm{N})>20)\end{array}$ & $\begin{array}{l}\quad \text { M } \\
\quad \text { For } \\
\text { proposed } \\
\text { algorithm } \\
\text { where } \\
\mathrm{P} / \mathrm{N})>20 \text { ) }\end{array}$ & $\begin{array}{l}\quad \text { Q } \\
\quad \text { For } \\
\text { proposed } \\
\text { algorithm } \\
\text { where } \\
(\mathrm{P} N)>20)\end{array}$ & $\begin{array}{c}\text { P } \\
\text { where } \\
(\mathrm{P} / \mathrm{N})>200\end{array}$ & $\begin{array}{c}\text { M } \\
\text { For } \\
\text { proposed } \\
\text { algorithm } \\
\text { Where } \\
(\mathrm{P} / \mathrm{N})>200)\end{array}$ & $\begin{array}{c}\text { Q } \\
\text { For } \\
\text { proposed } \\
\text { algorithm } \\
\text { where } \\
(\mathrm{P} / \mathrm{N})>200)\end{array}$ \\
\hline 5 & 3 & 0 & $00.00 \%$ & 11 & 2 & $66.66 \%$ & 101 & 3 & $100 \%$ & 1009 & 3 & $100 \%$ \\
\hline 7 & 5 & 0 & $00.00 \%$ & 17 & 2 & $40 \%$ & 149 & 5 & $100 \%$ & 1409 & 5 & $100 \%$ \\
\hline 11 & 9 & 0 & $00.00 \%$ & 23 & 1 & $11.11 \%$ & 223 & 8 & $88.88 \%$ & 2203 & 9 & $100 \%$ \\
\hline 13 & 11 & 0 & $00.00 \%$ & 29 & 1 & $9.09 \%$ & 263 & 11 & $100 \%$ & 2609 & 11 & $100 \%$ \\
\hline 17 & 15 & 0 & $00.00 \%$ & 37 & 4 & $26.66 \%$ & 347 & 14 & $93.33 \%$ & 3407 & 15 & $100 \%$ \\
\hline 19 & 17 & 0 & $00.00 \%$ & 41 & 4 & $23.52 \%$ & 383 & 15 & $88.23 \%$ & 3803 & 17 & $100 \%$ \\
\hline 23 & 21 & 0 & $00.00 \%$ & 47 & 3 & $14.28 \%$ & 461 & 18 & $85.71 \%$ & 4603 & 21 & $100 \%$ \\
\hline 29 & 27 & 0 & $00.00 \%$ & 59 & 11 & $40.74 \%$ & 587 & 23 & $85.18 \%$ & 5801 & 27 & $100 \%$ \\
\hline 31 & 29 & 0 & $00.00 \%$ & 67 & 6 & $20.68 \%$ & 631 & 28 & $96.55 \%$ & 6203 & 29 & $100 \%$ \\
\hline 37 & 35 & 0 & $00.00 \%$ & 79 & 5 & $14.28 \%$ & 743 & 33 & $94.28 \%$ & 7411 & 34 & $97.14 \%$ \\
\hline 41 & 39 & 0 & $00.00 \%$ & 83 & 14 & $35.89 \%$ & 821 & 38 & $97.43 \%$ & 8209 & 39 & $100 \%$ \\
\hline 43 & 41 & 0 & $00.00 \%$ & 89 & 4 & $9.75 \%$ & 863 & 37 & $90.24 \%$ & 8609 & 40 & $97.56 \%$ \\
\hline 47 & 45 & 0 & $00.00 \%$ & 97 & 10 & $22.22 \%$ & 941 & 45 & $100 \%$ & 9403 & 45 & $100 \%$ \\
\hline 53 & 51 & 0 & $00.00 \%$ & 107 & 17 & $33.33 \%$ & 1061 & 50 & $98.03 \%$ & 10601 & 50 & $98.03 \%$ \\
\hline 59 & 57 & 0 & $00.00 \%$ & 127 & 9 & $15.78 \%$ & 1181 & 55 & $96.49 \%$ & 11801 & 57 & $100 \%$ \\
\hline 61 & 59 & 0 & $00.00 \%$ & 127 & 9 & $15.25 \%$ & 1223 & 49 & $83.05 \%$ & 12203 & 58 & $98.30 \%$ \\
\hline 67 & 65 & 0 & $00.00 \%$ & 137 & 13 & $20 \%$ & 1361 & 57 & $87.69 \%$ & 13411 & 65 & $100 \%$ \\
\hline 71 & 69 & 0 & $00.00 \%$ & 149 & 11 & $15.94 \%$ & 1423 & 65 & $94.2 \%$ & 14207 & 66 & $95.65 \%$ \\
\hline 73 & 71 & 0 & $00.00 \%$ & 149 & 12 & $16.9 \%$ & 1471 & 61 & $85.91 \%$ & 14621 & 71 & $100 \%$ \\
\hline 79 & 77 & 0 & $00.00 \%$ & 163 & 17 & $22.07 \%$ & 1583 & 64 & $83.11 \%$ & 15803 & 77 & $100 \%$ \\
\hline 83 & 81 & 0 & $00.00 \%$ & 167 & 15 & $18.51 \%$ & 1663 & 69 & $85.15 \%$ & 16603 & 81 & $100 \%$ \\
\hline 89 & 87 & 0 & $00.00 \%$ & 179 & 29 & $33.33 \%$ & 1783 & 82 & $94.25 \%$ & 17807 & 86 & $98.85 \%$ \\
\hline 97 & 95 & 0 & $00.00 \%$ & 197 & 14 & $14.74 \%$ & 1949 & 82 & $86.31 \%$ & 19403 & 92 & $96.84 \%$ \\
\hline \multicolumn{3}{|c|}{ Average ratio } & $00.00 \%$ & & & $23.51 \%$ & & & $91.91 \%$ & & & $99.23 \%$ \\
\hline
\end{tabular}




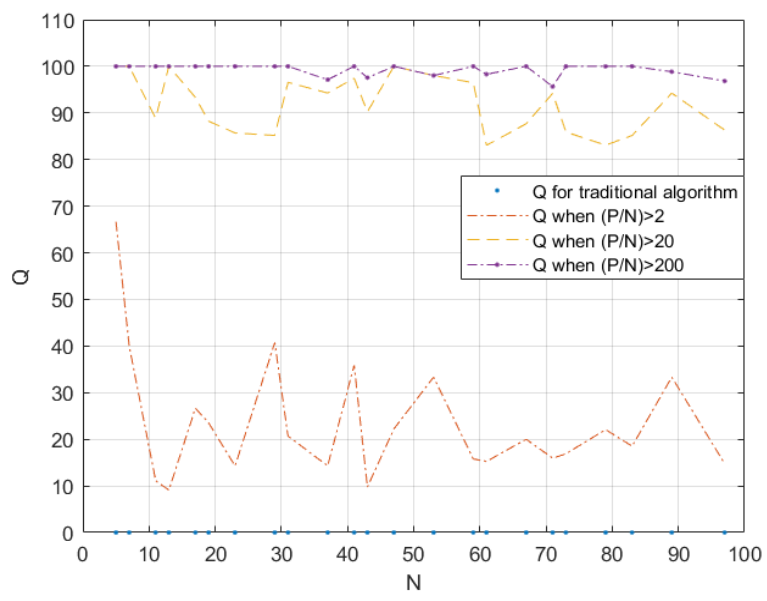

Figure 8. The relationship between $(\mathrm{Q}, \mathrm{N})$

\section{Extra Notes}

1-In the Chebyshev map $\left(T_{n}(x) \bmod N\right)$ any value for $\mathrm{N}$, which is Prime can be used in the Kocarev algorithm but this gives multiple keys problem as [8] said "Even if $\mathrm{N}$ is properly selected, the algorithm is still insecure from a practical viewpoint".

2- When $(\mathrm{n}=0)$ then $\left(T_{n}(x) \bmod H=1\right.$ for all values of $\mathrm{x}$ and that makes cipher text equal to plain text so we don't choose (s) or (r) equal to zero, where $\mathrm{H}$ is $\mathrm{N}$ or $\mathrm{P}$.

3- When $(\mathrm{x}=1)$ then $\left(T_{n}(x) \bmod H=1\right.$ for all values of $\mathrm{n}$ and that makes cipher text equal to plain text so we don't choose $(\mathrm{x}=1)$, where $\mathrm{H}$ is $\mathrm{N}$ or P.

4- When $(\mathrm{x}=0)$ then the outputs of map $\left(T_{n}(x) \bmod H\right)$ will be one of these values $(0,1$, $\mathrm{H}-1)$ of all values of (n) so we don't choose $(\mathrm{x}=0)$, where $\mathrm{H}$ is $\mathrm{N}$ or $\mathrm{P}$.

5- If $\left(T_{n}(x) \bmod H\right)=0$ for one value of $(\mathrm{n})$ or more we must not choose this value of (n) as (s or r) because:

A) This causes $T_{s}\left(T_{r}(x) \bmod H\right) \bmod H=$ $T_{r}\left(T_{s}(x) \bmod H\right) \bmod H=0$. That makes cipher text equal to zero and we cannot restore the plain text (in both algorithms), where $\mathrm{H}$ is $\mathrm{N}$ or $\mathrm{P}$.

B) If the result of $T_{s}\left(T_{r}(x) \bmod H\right) \bmod H=$ $T_{r}\left(T_{s}(x) \bmod H\right) \bmod H=1$ That makes cipher text equal to plain text (in both algorithms), where $\mathrm{H}$ is $\mathrm{N}$ or $\mathrm{P}$.

6-If $T_{n}(x) \bmod H=1$ for one value of (n) or more we must not choose this value of (n) as (s or $r$ ), because that causes $T_{s}\left(T_{r}(x) \bmod H\right) \bmod H=$ $T_{r}\left(T_{s}(x) \bmod H\right) \bmod H=1$ That makes cipher text equal to plain text (in both algorithms), where $\mathrm{H}$ is $\mathrm{N}$ or $\mathrm{P}$.

7-If $T_{n}(x) \operatorname{modH}=\mathrm{H}-1$ for one value of $(\mathrm{n})$ or more we must not choose this value of (n) as (s or $\mathrm{r}$ ), because that may cause $T_{s}\left(T_{r}(x) \bmod H\right) \bmod H=T_{r}\left(T_{s}(x) \bmod H\right) \bmod H=$ 1. That makes cipher text equals to plain text (in both algorithms), where $\mathrm{H}$ is $\mathrm{N}$ or $\mathrm{P}$.

\section{Conclusions}

In this paper, we introduce an improved public key cryptosystem based on Chebyshev map over finite field with ability to overcome the multiple keys problem i.e. ((there is only one key that can decrypt the system)). The proposed algorithm modified Kocarev algorithm by adding a new parameter $(\mathrm{P})$ that must be prime and it's value is greater than $\mathrm{N}$ and replacing (modP) instead of $(\operatorname{modN})$ in the proposed algorithm keeping $(s, r)$ less than $\mathrm{N}$. The results show that the proposed algorithm succeeds to remove the repetition problem completely as the ratio $(\mathrm{P} / \mathrm{N})$ increases and makes the system have only one key for decryption. This makes it more difficult to attack by third party.

\section{Conflict of interest}

The authors confirm that the publication of this article cause no conflict of interest. 


\section{References}

1. Shannon, C.E. (1949). Communication theory of secrecy systems. Bell System Technical Journal, Vol.28, No.4, PP. 656-715.

2. Shannon, C.E. (1948). A Mathematical Theory of Communication. Bell System Technical Journal, Vol.27, No.3, PP. 379-423.

3. Diffie, W., Hellman, M.E. (1976). New directions in cryptography. IEEE Transactions on Information Theory, Vol.22, No.6, PP 644-654.

4. Kocarev L. (2001). Chaos-based cryptography: A brief overview. IEEE Circuits Systems Magazine, Vol.1, No.3, PP.6-21.

5. L Kocarev, Z Tasev (2003)."Public-key encryption based on Chebyshev maps". ISCAS. IEEE international symposium on circuits systems, Bangkok, PP. 28-31.

6. P Bergamo, D'Arco P, De Santis A, Kocarev L (2005). Security of public-key encryption based on Chebyshev polynomials. IEEE Transactions on Circuits and Systems, Vol.52, No.7, PP.1382- 1393.

7. L Kocarev, J Makraduli, P Amato. (2005). Public-Key Encryption Based on Chebyshev Polynomials. Circuits, Systems and Signal Processing, Vol.24, No.5, PP.497-517.

8. Xiaofeng Liao, Fei Chen, and Kwok- Wo Wong (2010). On the Security of Public-Key Algorithms Based on Chebyshev Polynomials over the Finite Field ZN. IEEE Transactions on Computers, Vol. 59, No. 10, PP.13921401.

9. Jinhui Sun, Geng Zhao, Xufei Li. (2013). An Improved Public Key Encryption Algorithm Based on Chebyshev Polynomialsl. Telkomnika, Vol. 11, No. 2, PP.864-870.

10. Nedal Tahat, Emad Abdallah (2018). Hybrid Publicly Verifiable Authenticated Encryption Scheme Based on Chaotic Maps and
Factoring Problems. Journal of Applied Security Research, Vol. 13, No. 3, pp. 304-314. 